\title{
REVIEWS
}

\section{Association Between the Frequent Use of Perineal Talcum Powder Products and Ovarian Cancer: a Systematic Review and Meta-analysis}

\author{
Sean A Woolen, MD MSc ${ }^{7}$ (D) , Ann A. Lazar, $P h D^{2,3}$, and Rebecca Smith-Bindman, MD ${ }^{3,4,5}$ \\ 'Department of Radiology and Biomedical Imaging, University of California San Francisco, San Francisco, CA, USA; ${ }^{2}$ Department of Preventive and \\ Restorative Dental Sciences, University of California San Francisco, San Francisco, USA; ${ }^{3}$ Department of Epidemiology and Biostatistics, University of \\ California San Francisco, San Francisco, USA; ${ }^{4}$ Department of Obstetrics, Gynecology and Reproductive Sciences, University of California San \\ Francisco, San Francisco, USA; ${ }^{5}$ Philip R Lee Institute for Health Policy Studies, University of California San Francisco, San Francisco, USA.
}

BACKGROUND: Risk of ovarian cancer in women with frequent perineal talcum powder product is not well understood. Prior systematic reviews focused on ever use. The purpose of this study is to estimate the association between frequent (at least 2 times per week) perineal talcum powder use and ovarian cancer.

METHODS: A systematic review and meta-analysis was conducted according to meta-analysis of observational studies in epidemiology guidelines. Study protocol was prospectively registered at PROSPERO (registration number CRD42020172720). Searches were performed in PubMed, Embase, Web of Science, and Cochrane Central Register of Controlled Trials databases from their inception to August 2, 2021. Case-control and cohort studies were included if they reported frequent perineal talcum powder use and an adjusted odds ratio or hazard ratio for ovarian cancer. Review for inclusion, data extraction, and quality assessment (using the Newcastle-Ottawa Scale [NOS]) were performed independently by two reviewers. Pooled adjusted odds ratios with $95 \%$ confidence intervals were generated from the random effects model. Heterogeneity was quantified with $I^{2}$ statistic. Funnel plot and Eger's test were performed to assess publication bias. Subgroup and sensitivity analyses were performed for testing the robustness of the overall findings.

RESULTS: Initial database searches returned 761 unique citations and after review, eleven studies describing 66,876 patients, and 6542 cancers were included (Cohen's $\kappa=0.88$ ). Publication quality was high (median $\mathrm{NOS}=8$, range: 4 to 9 ). Frequent talcum powder use was associated with an elevated risk of ovarian cancer (adjusted pooled summary odds ratio 1.47 (95\% CI 1.31, 1.65, $P<0.0001)$. There was no evidence of bias and low heterogeneity $\left(I^{2}=24 \%, P=0.22\right)$. There was no meaningful difference limiting analysis to publications with a NOS quality score of 8 or 9 or limiting studies based on study design.

CONCLUSIONS: This review suggests an increased risk of ovarian cancer associated with frequent perineal powder exposure of $31-65 \%$.

Received September 14, 2021

Accepted January 13, 2022

Published online February 2, 2022
KEY WORDS: talcum powder; ovarian neoplasm; women's health; metaanalysis.

$\mathrm{J}$ Gen Intern Med 37(10):2526-32

DOI: $10.1007 / \mathrm{s} 11606-022-07414-7$

(c) The Author(s) 2022

\section{INTRODUCTION}

Talc, the primary ingredient in baby powder, is a naturally occurring mineral known for its softness and absorbency and has been added to a broad range of personal care products since the early part of the twentieth century. To date, there have been seven systematic reviews and pooled data studies ${ }^{1-7}$ of the relationship between talcum powder products and ovarian cancer. All studies including retrospective case-control studies have found a positive association. ${ }^{2-7}$ The most recent review by $\mathrm{O}^{\prime}$ Brien ${ }^{1}$ limited analysis to the four prospective cohort studies and in its main conclusion stated there was no statistically significant association between genital talc use and ovarian cancer.

The differences in conclusions between the metanalyses are at least partially due to the inconsistent talcum-based powder exposure questions regarding the frequency and type of exposure. To harmonize the exposure measurements across the greatest number of studies, prior meta-analyses primarily focused on quantifying the association between ever versus never use of talcum powder products and ovarian cancer. However, ever use is a non-specific exposure that could dilute or obscure a meaningful association as ever use would combine women with low and high exposures to talcum powder.

Knowledge of ovarian cancer risks are important for women's health and important to guide regulatory oversight. The purpose of this analysis is to estimate the risk of ovarian cancer associated with the frequent use of talcum powder products. We hypothesize that assessment of ovarian cancer risk among frequent users of talcum powder products would provide a more meaningful assessment of its carcinogenicity. 


\section{METHODS}

We conducted a systematic review of the literature and metaanalysis to assess the association between the frequent perineal exposure to talc and ovarian cancer following the Metaanalyses of Observational studies (MOOSE) reporting guidelines. A study protocol was developed in advance and registered with the International Prospective Register of Systematic Reviews (PROSPERO) on April 28, 2020 (registration number: CRD42020172720).

\section{Search Strategy and Information Sources}

Comprehensive searches were performed by an expert health science informationist from inception of the relevant databases to August 2, 2021. Searches were completed in the following: PubMed, Embase, Web of Science, and Cochrane Central Register of Controlled Trials. Each search consisted of talcum powder and ovarian cancer concept blocks, which can be viewed in the supplemental material. No date, language, or other restrictions were incorporated into the searches. Duplicate citations were removed in Endnote X9.3.1 (Clarivate Analytics). The references of all publications were searched to identify additional publications.

\section{Eligibility Criteria and Study Selection}

Selection of studies included observational cohort and casecontrol study designs. Studies were included if they reported primary data on frequent, defined as multiple ( 2 or more) times per week perineal exposure to talc, and reported an adjusted odds ratio or hazard ratio and $95 \%$ confidence interval (CI) for ovarian malignancy. Other uses of talcum powder, including applications to the body, were excluded to isolate exposure mechanism to perineal application. Conference abstracts, retracted manuscripts, narrative reviews, editorials, case reports, and manuscripts not reporting the location or frequency of talcum powder application were excluded.

Studies were screened for inclusion using prespecified selection criteria by a single author (SW). Selection criteria included publication of primary data, reporting on multiple times per week ( $\geq 2$ times per week) perineal exposure to talcum powder including direct application of talcum powder to the perineum and rectum, application to underwear or sanitary napkins, or on birth control devices like diaphragms and risk for ovarian malignancy. Studies were also selected for baseline quality requiring a multivariable risk adjustment, study size ( $n>10$ cancers), and defined research methods to allow assessment of inclusion and exclusion criteria (flow diagram Fig. 1). The search resulted in 761 citations, which were screened at the title and abstract level by a single author (SW). The full manuscripts of relevant citations $(n=52)$ were independently reviewed by two authors (SW and RSB). Agreement for inclusion was calculated using the Cohen $k$ with the following scale: 0.01 to 0.20 indicates slight; 0.21 to 0.40 , fair; 0.41 to 0.60 , moderate; 0.61 to 0.8 , substantial; and
0.81 to 0.99 , almost perfect. Disagreements were resolved by discussions.

The four prospective cohort studies as reported in $\mathrm{O}^{\prime} \mathrm{Brien}^{1}{ }^{\mathrm{did}}$ not meet the pre-specified definition for frequent exposure. However, the questionnaires for two of the included cohorts had asked women about more frequent talcum powder use. As is standard in systematic reviews to include relevant but unpublished results, we contacted O'Brien and requested primary data from the Nurses' Health Study 1 (NHS1) and the Sisters Study (SIS) for the highest frequency talc exposure group. The data from NHS 1 were provided and described in the Supplemental Table 1 and are included in the systematic review. The data from the SIS study were not provided to us due to the small sample size of exposed individuals in the highest exposure category ( $n=2$ women).

\section{Data Extraction}

Data extraction was performed by two authors (SW and RSB). Extracted information included age range, enrollment period, definition of frequency of talc use, size of control group or cohort size, number of ovarian cancers, and adjusted hazard ratio and odds ratio with $95 \%$ CI. Data were included from the highest reported talc use category to obtain as close to daily use as possible and the referent group were women who reported no talc exposure. When duplicate reports of the same subjects were published, the publication reporting the highest talc use was selected. The senior author was contacted to obtain data for studies that reported frequent perineal talcum powder exposure but did not publish the results on this exposure. Disagreements in data extraction were resolved by consensus.

\section{Assessment of Risk of Bias and Study Quality}

Eleven articles met selection criteria. The articles were independently reviewed by two authors (SW and RSB) for quality using the Newcastle Ottawa Scale (NOS) where a score of 0-3 reflects a very high risk of bias, 4-6 reflects a high risk of bias, and 7-9 reflects a high-quality study with low risk of bias. The scale assesses the criteria used to select study groups, the comparability of study groups, and ascertainment exposure or outcome of interest. Results of the validity assessment were discussed until agreement was reached (Table 1).

\section{Data Synthesis}

Individual study results were combined using summary estimates generated from the random effects model ${ }^{8}$ and displayed with forest plots. Adjusted odds ratios and the adjusted hazard ratio were combined given the infrequency of ovarian cancer. ${ }^{9}$ As a sensitivity analysis, we removed the one cohort study from the analysis and a study that combined nonperineal talcum powder users with perineal talcum powder users. 


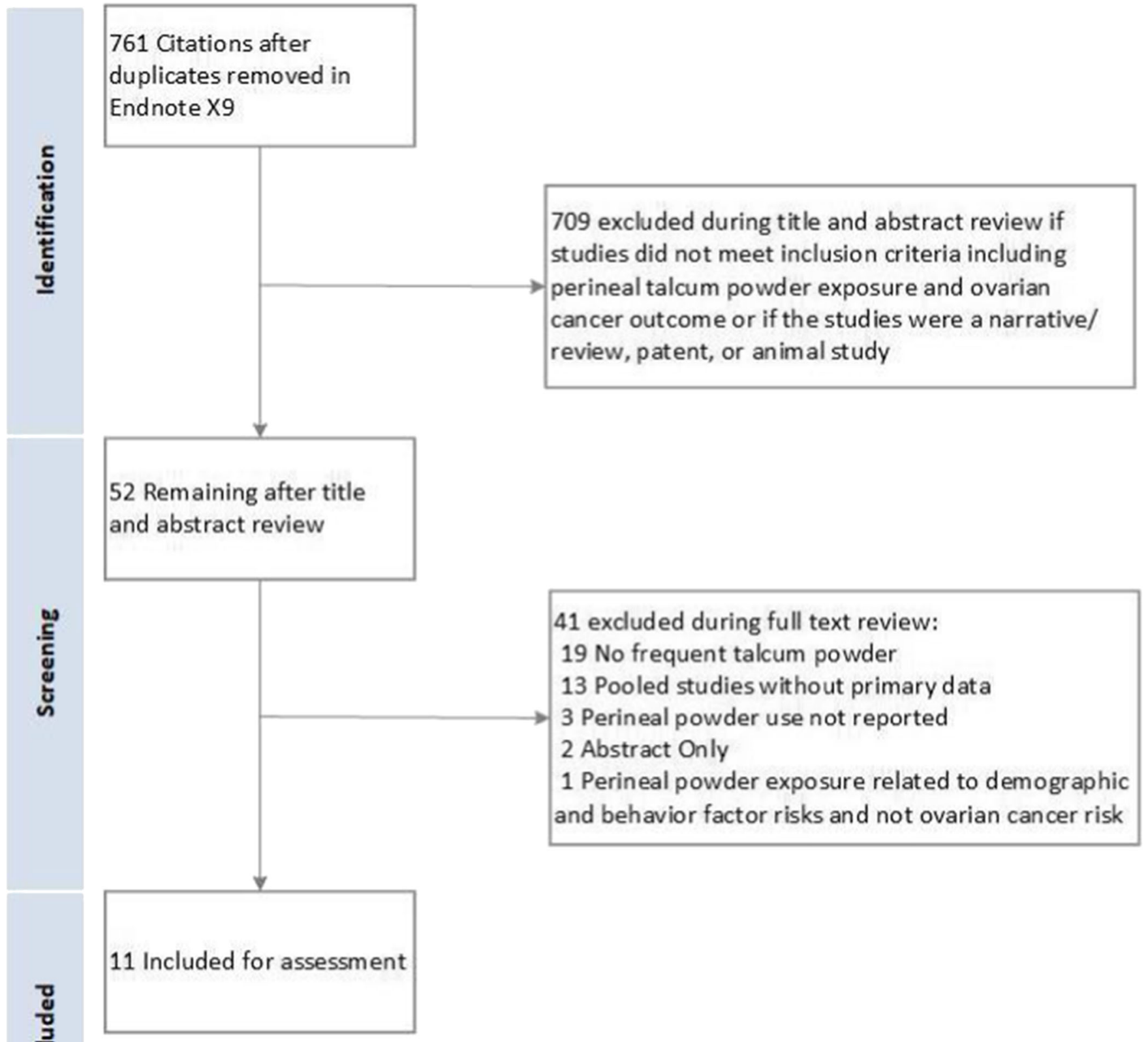

Figure 1. Flow diagram of study selection.

Heterogeneity was evaluated by inspecting funnel plots, and by calculating the $I^{2}$ values. Publication bias was evaluated by visual inspection of the funnel plot and statistically with Egger's test. The NOS score was calculated for each study and median, maximum, and minimum scores were calculated across all included studies. As sensitivity analyses, we repeated the meta-analysis first excluding the study with the NOS score of 4 , and then repeated the analysis limited to studies with an NOS score of 8 or 9. SAS v. 9.4 was used, and two-sided $P$-values less than 0.05 were considered statistically significant.

\section{RESULTS}

Initial database searches returned 761 unique citations (Fig. 1). After title and abstract review, 52 potential citations remained. After full-text review, 11 publications met the inclusion criteria including a total of 6542 ovarian cancer cases and 66,876 women (Table 2). ${ }^{1,10-19}$ The interrater agreement in determining the final study cohort from the 52 full-text reviews was excellent (Cohen's $k=0.88$ ). The included studies include 10 retrospective case-control studies and a single cohort 
Table 1. Quality Assessment of Included Studies Using the Newcastle-Ottawa Scale

\begin{tabular}{|c|c|c|c|c|}
\hline Case-control design* & Selection & Comparability & Exposure & Total score (out of 9 ) \\
\hline Booth et al., $1989^{10}$ & $* *$ & $* *$ & - & 4 \\
\hline Chang et al., 199 [11] & $* * * *$ & $* *$ & $* *$ & 8 \\
\hline Cook et al., $1997^{12}$ & $* * * *$ & $*$ & $* *$ & 7 \\
\hline Cramer et al., $2016^{13}$ & $* * * *$ & $* *$ & $* *$ & 8 \\
\hline Harlow et al., $1992^{14}$ & $* * * *$ & $* *$ & $* *$ & 8 \\
\hline Mills et al., $2004^{15}$ & $* * * *$ & $* *$ & $* *$ & 8 \\
\hline Rosenblatt et al., $2011^{16}$ & $* * * *$ & $* *$ & $* *$ & 8 \\
\hline Schildkraut et al., $2016^{17}$ & $* * * *$ & $* *$ & $* *$ & 8 \\
\hline Whittenmore et al., $1988^{18}$ & $* * *$ & $* *$ & $* *$ & 7 \\
\hline Wu et al., $2009^{19}$ & $* * * *$ & $* *$ & $* *$ & 8 \\
\hline Cohort design & & & Outcome & \\
\hline O’Brien (National Heath Study 1), $2020^{1}$ & $* * * *$ & $* *$ & $* * *$ & 9 \\
\hline
\end{tabular}

Each asterisk denotes 1 point. The empty cells indicate the study received no points in the category.

All the case-control studies lost a point in the exposure category because they did not report if the interviewers were blinded to cancer status when the interviews were conducted.

study. The median NOS of included case-control studies was 8 (range: 4 to 8 ). The NOS of the included cohort study was 9.

The age range of included women was 18-79 years. Studies were published between 1988 and 2016. Among included studies, the range of frequent talcum powder use was defined as 4-7× per week, and 45\% (5 of 11) reported daily exposure. The studies were homogeneous, and $24.4 \%(P=0.22)$ of the variation across studies were due to heterogeneity. The summary pooled odds ratio assessing the association between frequent

Table 2. Publications Included in the Systematic Review. The Most Frequent Perineal Talcum Powder Use Reported for Each Study Was Abstracted

\begin{tabular}{|c|c|c|c|c|c|c|c|c|c|c|}
\hline & \multirow[t]{2}{*}{ First author } & \multirow[t]{2}{*}{$\begin{array}{l}\text { Study } \\
\text { type }\end{array}$} & \multirow[t]{2}{*}{ Year } & \multirow[t]{2}{*}{$\begin{array}{l}\text { Age } \\
\text { range }\end{array}$} & \multirow[t]{2}{*}{ Enrollment period } & \multirow[t]{2}{*}{$\begin{array}{l}\text { Specification of } \\
\text { talc exposure }\end{array}$} & \multicolumn{2}{|c|}{$\begin{array}{l}\text { Ovarian cancer } \\
\text { cases }\end{array}$} & \multicolumn{2}{|c|}{ Controls or cohort } \\
\hline & & & & & & & $\begin{array}{l}\text { No. } \\
\text { Exposed }\end{array}$ & $\begin{array}{l}\text { No. } \\
\text { Total }\end{array}$ & $\begin{array}{l}\text { No. } \\
\text { Exposed }\end{array}$ & $\begin{array}{l}\text { No. } \\
\text { Total }\end{array}$ \\
\hline 1 & Booth $\mathrm{M}^{10}$ & $\begin{array}{l}\text { Case- } \\
\text { control }\end{array}$ & 1989 & $20-64$ & $1978-1983$ & Daily & 71 & 217 & 139 & 434 \\
\hline 2 & Chang $\mathrm{S}^{11}$ & $\begin{array}{l}\text { Case- } \\
\text { control }\end{array}$ & 1997 & $35-79$ & 1989-1992 & $>25 \times$ per month & 41 & 450 & 60 & 564 \\
\hline 3 & Cook LS ${ }^{12(1)}$ & $\begin{array}{l}\text { Case- } \\
\text { control }\end{array}$ & 1997 & $20-79$ & 1986-1988 & $>10,000$ lifetime & 28 & 313 & 17 & 422 \\
\hline 4 & Cramer DW ${ }^{13}$ & $\begin{array}{l}\text { Case- } \\
\text { control }\end{array}$ & 2016 & $18-80$ & $\begin{array}{l}1992-19971995- \\
20022003-2008\end{array}$ & $>30 \times$ per month & 267 & 2041 & 205 & 2100 \\
\hline 5 & Harlow $\mathrm{BL}^{14(2)}$ & $\begin{array}{l}\text { Case- } \\
\text { control }\end{array}$ & 1992 & $18-76$ & $1984-1987$ & $>10,000$ lifetime & 58 & 235 & 41 & 239 \\
\hline 6 & Mills $\mathrm{PK}^{15(1)(3)}$ & $\begin{array}{l}\text { Case- } \\
\text { control }\end{array}$ & 2004 & $41-70$ & $2000-2001$ & $4-7 \times$ per week & 41 & 249 & 122 & 1100 \\
\hline 7 & $\begin{array}{l}\text { Rosenblatt } \\
\mathrm{KA}^{16(1)}\end{array}$ & $\begin{array}{l}\text { Case- } \\
\text { control }\end{array}$ & 2011 & $35-74$ & $2002-2005$ & $>10,000$ lifetime & 18 & 812 & 37 & 1313 \\
\hline 8 & $\begin{array}{l}\text { Schildkraut } \\
\mathrm{JM}^{17(1)(3)}\end{array}$ & $\begin{array}{l}\text { Case- } \\
\text { control }\end{array}$ & 2016 & $20-79$ & 2010-2015 & Daily & 158 & 582 & 134 & 744 \\
\hline 9 & $\begin{array}{l}\text { Whittemore } \\
\mathrm{AS}^{18}\end{array}$ & $\begin{array}{l}\text { Case- } \\
\text { control }\end{array}$ & 1988 & $18-74$ & 1983-1985 & $>20 \times$ per month & 44 & 188 & 101 & 539 \\
\hline 10 & $\mathrm{Wu} \mathrm{AH}^{19(4)}$ & $\begin{array}{l}\text { Case- } \\
\text { control }\end{array}$ & 2009 & $18-74$ & 1998-2002 & $\begin{array}{l}>30 \times \text { per month } \\
\text { and }>20 \text { years }\end{array}$ & 67 & 605 & 45 & 688 \\
\hline 11 & $\begin{array}{l}\text { O’Brien (NHS } \\
1)^{1(5)}\end{array}$ & Cohort & 2020 & $35-62$ & 1982-2016 & Daily & 157 & 850 & 355 & 52191 \\
\hline
\end{tabular}

NHS Nurses' Health Study

For each study that specified the number of women who did not respond to talc questions, these women were subtracted from the total number of cases and controls.

${ }^{(1)}$ Cook, Mills, Rosenblatt, and Schildkraut did not differentiate between talc and cornstarch powders. Cornstarch is estimated to reflect $1-2 \%$ of powder

${ }^{(2)}$ Harlow reports an adjusted odds ratio for daily talcum powder exposure, and for $>10,000$ lifetime uses. The point estimates are the same for each and the 95\% CI almost identical. We include data for $>10,000$ lifetime uses as this number is explicitly defined as perineal exposure

${ }^{(3)}$ Shildkraut was the only study that included women recruited after two class action lawsuits were filed in 2014 concerning possible carcinogenic effects of body powder influencing recall of use. The study adjusted for individuals answering questions after 2014 to account for increased recall bias

${ }^{(4)}$ Wu combined non-perineal with perineal exposures. Wu reported an adjusted odds ratio for women who used talcum powder $>30 \times$ per month and $>$ 20 years

${ }^{(5)}$ O'Brien did not publish on daily exposure for the National Health Study participants. However, these data were available and O'Brien provided these data for inclusion. The entirety of the data we were provided are shared in the supplementary table. We include data on women with intact fallopian tubes, to harmonize with other publications 


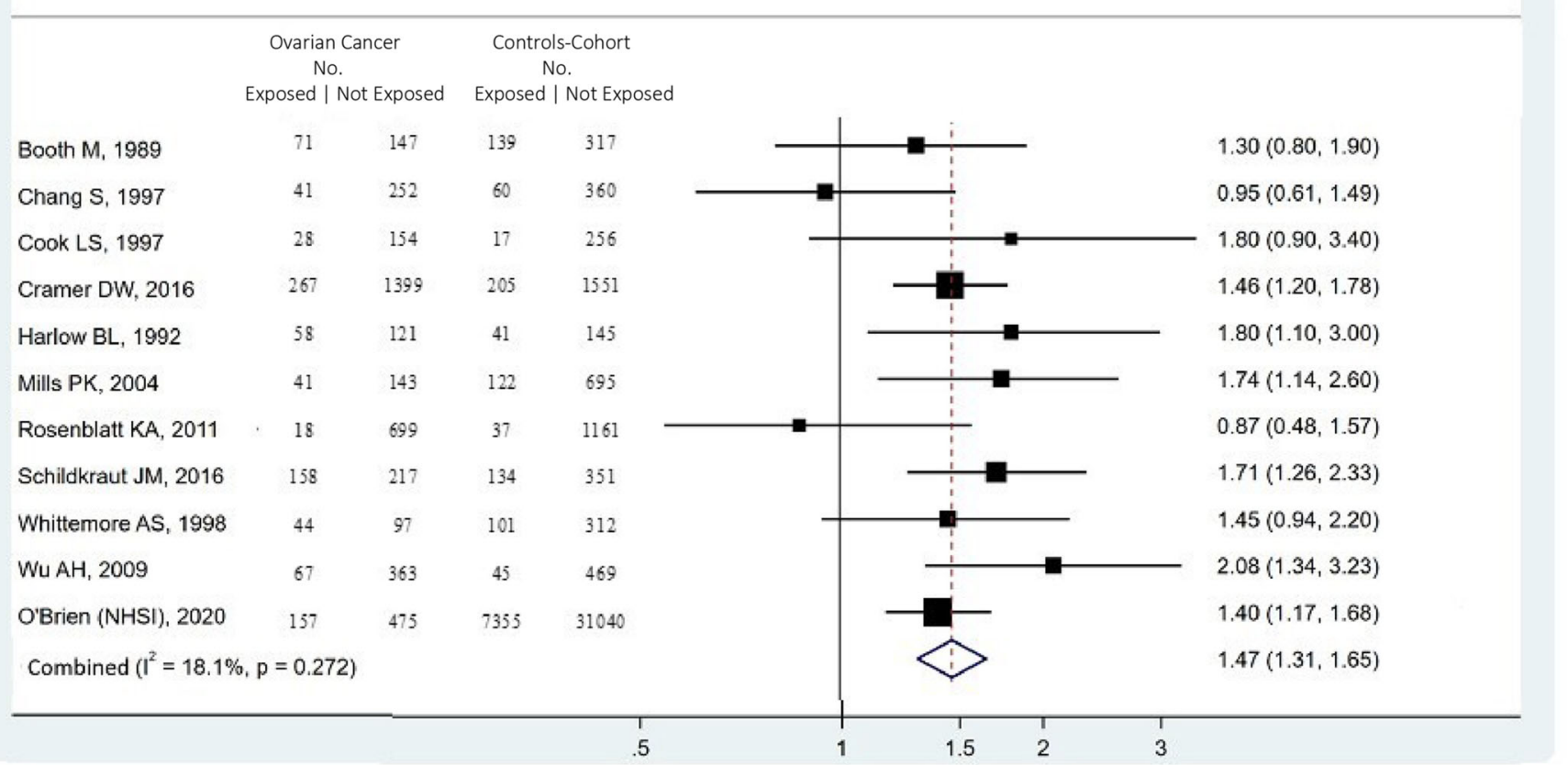

Figure 2. Forest plot showing the summary meta-analytic estimate for the association between frequent use of perineal talcum powder products and the risk of ovarian cancer. The number (No.) of women included as cases and controls (or cohort) who were exposed and not exposed are provided (excluding from the table women who had exposure to talcum powder at less than the highest exposure). The study-specific odds ratios and $95 \%$ confidence interval are on the right side of the plot.

use of perineal talcum powder products and ovarian cancer was $1.47(P<0.0001,95 \%$ CI 1.31, 1.65) (Fig 2) and there was no significant publication bias (Egger's test, $\mathrm{P}=0.94$ ) (Fig 3). When limited to the case-control studies, the summary pooled odds ratio was $1.49(P<0.0001,95 \%$ CI $1.29,1.72)$ (Supplementary Figures 1 and 2), whereas the odds ratio for the cohort study was 1.40 (95\% CI 1.17, 1.68). When excluding Wu et al. ${ }^{19}$ which combined perineal administration of talcum powder with other methods, the summary pooled odds ratio was $1.44(95 \%$ CI $1.29,1.60$ ) (Supplementary Figure 3 ) and the studies remained homogenous $\left(I^{2}=6.5 \%, P=0.382\right)$ without publication bias (Egger's test, $P=0.77$ ) (Supplementary Figure 4).

Given the quality of a meta-analysis is dependent on the quality of the studies it includes, we conducted a sensitivity analysis excluding the study with a NOS grade of $4\left(\right.$ Booth $\left.^{10}\right)$. There was no meaningful change after excluding Booth, summary pooled odds ratio $1.48(P<0.0001,95 \%$ CI 1.31 to 1.69$)$ (Supplementary Figure 5) and the studies remained homogenous $\left(I^{2}=24.4 \%, P=0.22\right)$ without publication bias (Egger's test, $P=0.88$ ) (Supplementary Figure 6). The second sensitivity analysis was restricted to studies with a NOS of 8 or 9 , removing Booth et al. ${ }^{10}$ (NOS=4), Cook et al. ${ }^{12}$ (NOS=7), and Whittemore et al. ${ }^{18}$ (NOS=7), also without a statistically significant change in the summary pooled odds ratio of $1.48(P<0.0001,95 \%$ CI 1.27 to 1.72 ) (Supplementary Figure 7$)$. The studies remained homogenous $\left(I^{2}=39.3 \%, P=0.12\right)$ and did not have publication bias (Egger's test, $P=0.97$ ) (Supplementary Figure 8).

\section{DISCUSSION}

We found frequent use of perineal talcum powder is associated with an increased risk of ovarian cancer, with a pooled adjusted odds ratio of 1.47 (95\% CI 1.31, 1.65). The 11 contributing studies included in the review, including the longest published follow-up available from the Nurses' Health Study cohort, were homogenous, and the summary estimate was robust in the sensitivity analysis. This meta-analysis makes a significant contribution to the available evidence as it is the first study that summarizes the published cohort and case-control literature that focuses on frequent (multiple times per week) rather than ever use of talc.

The precise mechanism whereby talcum powder causes ovarian cancer is not fully understood. It is widely speculated that trans-genital migration of talc powder through the fallopian tubes to the ovaries and peritoneum results in inflammation and a cascade of changes that result in carcinogenesis. ${ }^{20,21}$ Talc fibers are found within normal ovaries and within ovarian cancer with "cosmetic" talc use ${ }^{22,23}$ supporting this theory. A case series by Steffen et al. reported 10 cases of patients with "cosmetic" talc exposure and serous ovarian cancer evaluated the surgical specimens as well as cosmetic talcum powder 


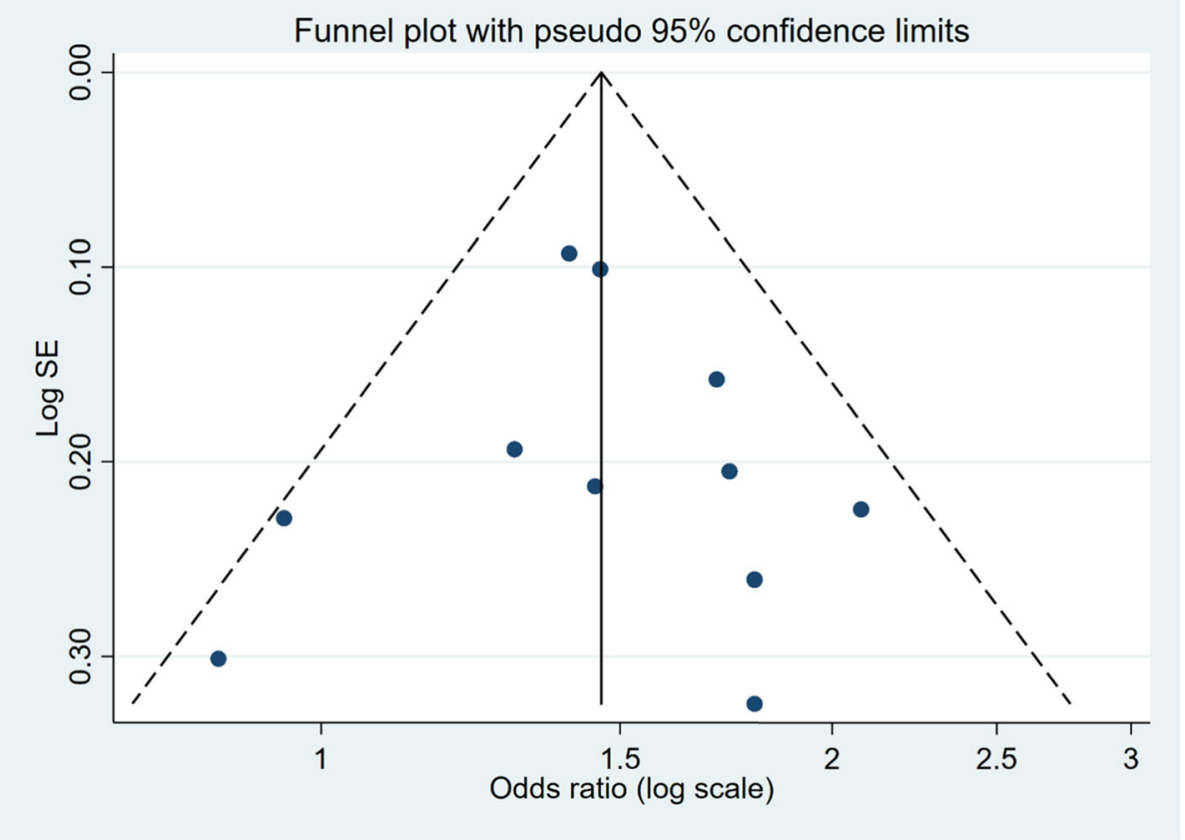

Figure 3. Funnel plot for the risk of publication bias.

manufactured in the 1950s. The transmission electron microscope tissue analysis and phase contrast microscopy found talc in every serous ovarian cancer and anthophyllite asbestos in $80 \%(8 / 10)$ of the serous ovarian cancers, which matched the talcum-based powder from the 1950s. Asbestiform talc is considered, along with asbestos, as a class 1 carcinogen for ovarian cancer, by the International Agency for Research on Cancer. ${ }^{24}$ Thus, the strongest and most widely believed theory is that asbestiform talc fibers and asbestos fibers are the etiological agents leading to the elevated cancer risk associated with their use.

The pooled odds ratio of 1.47 is higher than that of six additional systematic reviews which included case-control studies, all of which also report a statistically significant association between talc and ovarian cancer (range of adjusted odds ratios: $1.24-1.35) .^{2-7}$ O'Brien et al. ${ }^{1}$ concluded that perineal talcum powder exposure in cohort studies was not significantly associated with ovarian cancer. However, when O'Brien limited to women with patent fallopian tubes the hazard was 1.13 (95\% CI: 1.01 to 1.26 ) for ever versus never use and there was a dose response with increasing risk with increasing frequency of talcum powder use with a hazard ratio of 1.40 (95\% CI: 1.17 to 1.68 ) for daily users. The magnitude of the higher association in our study compared to prior casecontrol and cohort meta-analyses was likely due to our focus on frequent rather than any talcum powder users, inclusion of quality studies, and consistent definition of the exposure.

Our meta-analysis included both case-control and cohort observational studies allowing use to include more cancer cases than cohort alone. Although there is the general belief that cohort studies are better than case-control studies, both can provide accurate and meaningful information about statistical associations. Since most publications on talc used a case-control design with detailed quantification of the type and frequency of talc exposure missing from most of the cohort studies, it is important to include the data from the case-control studies in any summary estimate of the association with ovarian cancer. While cohort studies can provide a superior design for some research questions, the method provides less useful results when quantification of the exposure is too crude to provide a meaningful estimation of exposure. Cohort studies for talcum powder did not define the type of powder exposure, consistently define frequency and duration, capture changes in exposure over time, and relied on recall by participants for exposure which may involve misclassification as would be expected in case control studies. ${ }^{25-27}$ Although it is well known that case-control study design is susceptible to recall bias and misclassification, the bias was likely limited to participant recollection of their exposure rather than influenced by the media, given there was no publicity on the topic at the time of most publications: the first legal case was reported in the press in 2014 long after most studies completed recruitment. The one study where patient recruitment occurred after 2014 patients was Schildkraut et al., ${ }^{17}$ where recruitment occurred between 2010 and 1015, and they adjusted for the additional bias.

\section{Strengths and Limitations}

The primary strength of our study is our focus on frequent users of perineal talcum powder. Among women who report talcum powder use, the most common frequency is daily use, ${ }^{13}$ and this is the first systematic review to focus on multiple times per week users. The results were highly consistent and homogenous, and the included studies were of high quality. The work has limitations as well. We constructed our 
selection criteria prospectively to include studies with multiple times per week and as close to daily talcum powder exposure as possible. However, this meant that cohort and case-control studies that might have frequent-use patients were excluded if the questionnaire did not explicitly capture this information. The definition of talcum powder use varied by frequency and duration between the case-control and cohort studies. Additionally, studies by Cook et al. ${ }^{12}$, Mills et al. ${ }^{15}$, Rosenblatt et al. ${ }^{16}$, and Schildkraut et al. ${ }^{17}$ were unable to differentiate between use of perineal powders and the small subset using cornstarch (estimated at 1.5\%). However, the differences in definition and small inclusion of cornstarch likely did not affect the results as there was no evidence for statistical heterogeneity in our study. The included studies were adjusted for multiple covariates. The possibility of additional confounders to the studies likely exists.

\section{Conclusions and Implications}

In this analysis of pooled data from 10 case-control studies and a single cohort study, the frequent use of perineal talcum powder use is associated with increased risk of ovarian cancer. These results support women avoiding the frequent use of talcum powder in the perineal area.

Supplementary Information The online version contains supplementary material available at https://doi.org/10.1007/s11606-02207414-7.

Acknowledgements: We thank Evans Whitaker, MD MLIS, for his help in providing the literature review.

We thank Katie O'Brien, $P h D$, for her help in providing data from the SIS and NHS1 studies.

Corresponding Author: Sean A Woolen, MD MSc; Department of Radiology and Biomedical Imaging, University of California San Francisco, San Francisco, CA, USA (e-mail: sean.woolen@ucsf.edu).

\section{Declarations:}

Conflict of Interest: Sean Woolen: No relevant disclosures. Ann A Lazar: No relevant disclosures.

Rebecca Smith-Bindman: Served as a paid expert witness for the plaintiffs in the talcum powder litigation.

\section{REFERENCES}

1. O'Brien KM, Tworoger SS, Harris HR, et al. Association of Powder Use in the Genital Area With Risk of Ovarian Cancer. Jama 2020;323(1):4959.

2. Gross AJ, Berg PH. A meta-analytical approach examining the potential relationship between talc exposure and ovarian cancer. $J$ Expo Anal Environ Epidemiol 1995;5(2):181-195.

3. Langseth H, Hankinson SE, Siemiatycki J, Weiderpass E. Perineal use of talc and risk of ovarian cancer. $J$ Epidemiol Community Health 2008;62(4):358-360
4. Terry KL, Karageorgi S, Shvetsov YB, et al. Genital Powder Use and Risk of Ovarian Cancer: A Pooled Analysis of 8,525 Cases and 9,859 Controls. Cancer Prevention Research 2013;6(8):811-821.

5. Berge W, Mundt $\mathbf{K}$, Luu $\mathbf{H}$, Boffetta $\mathbf{P}$. Genital use of talc and risk of ovarian cancer: a meta-analysis. Eur J Cancer Prev 2018;27(3):248-257.

6. Penninkilampi R, Eslick GD. Perineal Talc Use and Ovarian Cancer: A Systematic Review and Meta-Analysis. Epidemiology 2018;29(1):41-49.

7. Taher KM, Farhat N, Karyakina NA, et al. Critical review of the association between perineal use of talc powder and risk of ovarian cancer. Reproductive toxicology (Elmsford, NY) 2019;90:88-101.

8. DerSimonian R, Laird N. Meta-analysis in clinical trials. Control Clin Trials 1986;7(3): 177-188.

9. Cummings $\mathbf{P}$. The relative merits of risk ratios and odds ratios. Arch Pediatr Adolesc Med. Arch Pediatr Adolesc Med 2009; 163(5): 438-45.

10. Booth M, Beral V, Smith P. Risk factors for ovarian cancer: a casecontrol study. Br J Cancer 1989;60(4):592-598.

11. Chang S, Risch HA. Perineal talc exposure and risk of ovarian carcinoma. Cancer 1997;79(12):2396-2401.

12. Cook LS, Kamb ML, Weiss NS. Perineal powder exposure and the risk of ovarian cancer. Am J Epidemiol 1997; 145(5):459-465.

13. Cramer DW, Vitonis AF, Terry KL, Welch WR, Titus LJ. The Association Between Talc Use and Ovarian Cancer: A Retrospective Case-Control Study in Two US States. Epidemiolog 2016;27(3):334-346.

14. Harlow BL DC, Bell DA, Welch WR. Perineal exposure to talc and ovarian cancer risk. Obstet Gynecol 1992;80(1): 19-26.

15. Mills PK, Riordan DG, Cress RD, Young HA. Perineal talc exposure and epithelial ovarian cancer risk in the Central Valley of California. Int $J$ Cancer 2004; 112(3):458-464

16. Rosenblatt KA, Weiss NS, Cushing-Haugen KL, Wicklund KG, Rossing MA. Genital powder exposure and the risk of epithelial ovarian cancer. Cancer Causes Control 2011;22(5):737-742.

17. Schildkraut JM, Abbott SE, Alberg AJ, et al. Association between Body Powder Use and Ovarian Cancer: The African American Cancer Epidemiology Study (AACES). Cancer Epidemiol Biomarkers Prev 2016;25(10):1411-1417.

18. Whittemore AS, Wu ML, Paffenbarger Jr RS, et al. Personal and environmental characteristics related to epithelial ovarian cancer. II. Exposures to talcum powder, tobacco, alcohol, and coffee. American Journal of Epidemiology 1988;128(6):1228-1240.

19. Wu AH, Pearce CL, Tseng C-C, Templeman C, Pike MC. Markers of inflammation and risk of ovarian cancer in Los Angeles County. International Journal of Cancer 2009; 124(6): 1409-1415.

20. Savant SS, Sriramkumar S, O'Hagan HM, et al. The Role of Inflammatory Mediatorsin the Development, Progression, Metastasis, and Chemoesistance of Epithelial Ovarian Cancer. Cancers 2018;10(8):251.

21. Fletcher NM, Harper AK, Memaj I, Fan R, Morris RT, Saed GM. Molecular Basis Supporting the Association of Talcum Powder Use with Increased Risk of Ovarian Cancer. Reprod Sci. 2020; 27(10):1836-1838.

22. Henderson WJ, Hamilton TC, Grifiths $\mathbf{K}$. Talc in Normal and Malignant Ovarian Tissue. The Lancet 1979; 313: 499.

23. Steffen JE, Tran T, Yimam M, et al. Serous Ovarian Cancer Caused by Exposure to Asbestos and Fibrous Talc in Cosmetic Talc Powders-A Case Series. J Occup Environ Med 2020; 62(2):e65-e77.

24. World Health Organization. International Agency for Research on Cancer Monographs on the Identification of Carcinogenic Hazards to Humans. World Health Organization. https://monographs.iarc.fr/list-of-classifications. Published June 26, 2020. Accessed August 15, 2020.

25. Gossett DR, Del Carmen MG. Use of Powder in the Genital Area and Ovarian Cancer Risk: Examining the Evidence. JAMA 2020; 323(1): 2931.

26. Cramer DW. Genital Powder Use and Ovarian Cancer. JAMA 2020; 323 (20):2095-2096.

27. Harlow BL, Murray EJ, Rothman KJ. Genital Powder Use and Ovarian Cancer. JAMA 2020; 323(20): 2096.

Publisher's Note Springer Nature remains neutral with regard to jurisdictional claims in published maps and institutional affiliations. 